\title{
Confinement and Chiral Symmetry, a Lattice QCD test of AdS/QCD
}

\author{
D. K. Sinclair ${ }^{* \dagger}$ \\ HEP Division, Argonne National Laboratory, 9700 South Cass Avenue, Argonne, IL 60439, USA \\ E-mail: dks@hep.anl.gov
}

We use lattice QCD simulations to test some of the predictions of proposed AdS/QCD (holographic) duals for QCD. In particular, these duals predict that the scale of chiral symmetry breaking $(\chi \mathrm{SB})$ can be varied independently from that of confinement, with the proviso that the scale of $\chi \mathrm{SB}$ cannot be longer than that of confinement. We simulate lattice QCD with 2 quarks in the fundamental representation of colour and with additional 4-fermion interactions (suggested by AdS/QCD), at finite temperatures. For sufficiently strong 4-fermion interactions, the deconfinement and $\chi \mathrm{SB}$ transitions occur at different temperatures, the separation depending on the 4-fermion coupling. This confirms that the scales of confinement and $\chi \mathrm{SB}$ are, in general, different.

The XXVI International Symposium on Lattice Field Theory

July $14-19,2008$

Williamsburg, Virginia, USA

\footnotetext{
* Speaker.

${ }^{\dagger}$ This research was supported in part by US Department of Energy contract DE-AC02-06CH11357, and in part under a Joint Theory Institute grant.
} 


\section{Introduction}

With standard actions, simulations of the finite temperature phase structure of lattice QCD with fundamental quarks indicate that the deconfinement transition and the chiral-symmetry restoration transition are coincident [1]. For quarks in other representations of $S U(3)_{\text {colour }}$, deconfinement occurs at a lower temperature than chiral-symmetry restoration [2, 3].

On the other, hand suggested holographic duals of QCD with fundamental quarks, inspired by AdS/CFT duality [ 7 , 5, 母, predict that the scales of confinement and of chiral symmetry breaking $(\chi \mathrm{SB})$ can be varied independently [7, 8]. However, since confinement produces $\chi \mathrm{SB}$, the length scale associated with $\chi \mathrm{SB}$ cannot be greater than that associated with confinement [9] 10].

These proposed holographic (string/gravity) duals of QCD suggest that the scales of confinement and $\chi \mathrm{SB}$ can be decoupled by the addition of (non-local) 4-fermion interactions to QCD. Adding attractive 4-fermion interactions binds the quarks and antiquarks more tightly so that $q \bar{q}$ pairs can condense and spontaneously break chiral symmetry at shorter distances.

We consider lattice QCD with 2-flavours of staggered quarks and local 4-fermion interactions of the Gross-Neveu [11]/Nambu-Jona-Lasinio [12, 13] type. We work at finite temperature and use the deconfinement and chiral-symmetry restoration temperatures as our measure of the scales of confinement and $\chi \mathrm{SB}$. These are measured as functions of the 4 -fermion coupling. These simulations are described in reference [14]

\section{The lattice action}

The lattice quark action is the standard $\chi \mathrm{QCD}$ action [15], which is the traditional staggered quark action augmented by a chiral 4-fermion term. Expressed in terms of the auxilliary fields $\sigma$ and $\pi$ to render it quadratic in the fermion fields it is

$$
S_{f}=\sum_{f=1}^{N_{f} / 4} \sum_{s} \bar{\chi}_{f}\left[\not D+m+\frac{1}{16} \sum_{i}\left(\sigma_{i}+i \varepsilon \pi_{i}\right)\right] \chi_{f}+\sum_{\tilde{s}} \frac{1}{8} N_{f} \gamma\left(\sigma^{2}+\pi^{2}\right)
$$

$\varepsilon=(-1)^{x+y+z+t}$ and $\gamma$ is the inverse 4-fermion coupling. This preserves the exact $U(1)$ axial flavour symmetry of staggered fermions.

Simulations are performed using the exact RHMC algorithm [16] to tune to 2 flavours. The deconfinement transition is determined as the position of the rapid increase in the Wilson Line (Polyakov Loop). The chiral-symmetry restoration phase transition occurs where the chiral-condensate $\bar{\psi} \psi$ vanishes $(m=0)$.

\section{Simulations and Results}

If we turn off the QCD interactions, we are left with a 4-fermion model which has a bulk transition at $\gamma=\gamma_{c} \approx 1.7$. At finite temperature, this will be shifted to smaller $\gamma$ (stronger coupling).

Our finite temperature simulations are performed on $N_{t}=4$ lattices. We keep $\gamma>\gamma_{c}$ so that at high temperatures - weak gauge coupling (large $\beta=6 / g^{2}$ ) - the theory is in the chiral-symmetry restored phase. Previous simulations at $\gamma=10$ and $\gamma=20$ indicate that the deconfinement and 
chiral-symmetry restoring transitions are coincident [17]. Hence $\gamma \geq 10$ represents a weak 4fermion coupling. We simulate at $\gamma=2.5$, a strong 4-fermion coupling and $\gamma=5$, and intermediate coupling. $N_{f}=2$ and $m=0$. At $\gamma=2.5$ our lattice sizes are $16^{3} \times 4,24^{3} \times 4$ and $32^{3} \times 4$. At $\gamma=5$ our lattice sizes are $12^{2} \times 24 \times 4,24^{3} \times 4$ and $32^{3} \times 4$. Typical run lengths for each parameter set are 50,000 or 100,000 trajectories.

Figure 1 shows the Wilson Line and chiral condensate as functions of $\beta$ for the two $\gamma_{\mathrm{s}}$ considered. At $\gamma=2.5$ the deconfinement transition and the chiral-symmetry restoration transition are well separated. At $\gamma=5$ the two transitions are close, but still clearly separate. It is clear that the finite size effects are small near the deconfinement transition. As expected, the finite size effects are considerable close to the chiral-symmetry restoration transition. These graphs include points (and for $\gamma=2.5$ a lattice size) in addition to those in our earlier publication [14].
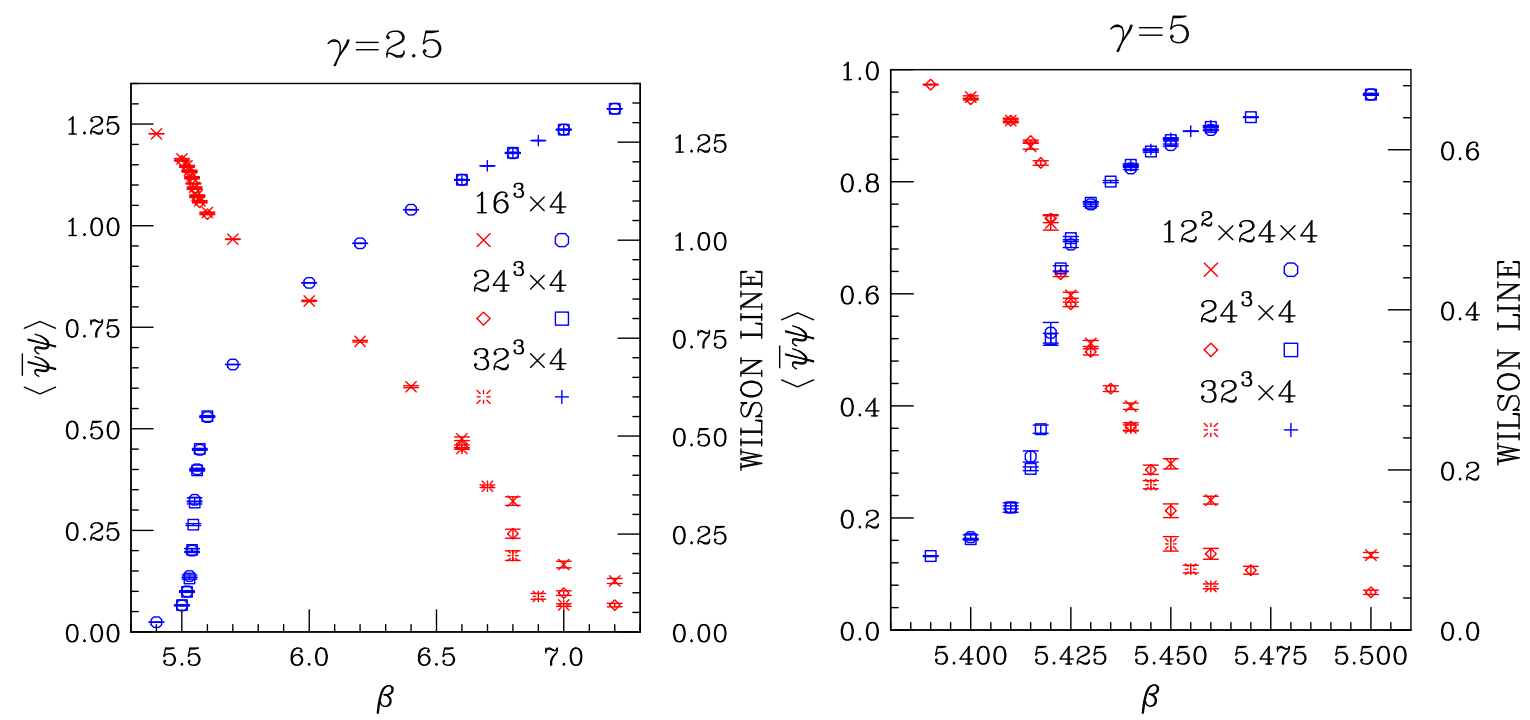

Figure 1: Wilson line and chiral condensate as functions of $\beta$ for (a) $\gamma=2.5$ and (b) $\gamma=5$ in lattice units.

At $\gamma=2.5$ we estimate the position of the deconfinement transition from the peak in the Wilson Line susceptibility, using Ferrenberg-Swendsen reweighting [18] from $\beta=5.545$. This yields $\beta_{d}=5.547(3)$. This is possible because the distributions of plaquette values from the simulated $\beta \mathrm{s}$ in the neighbourhood of this transition overlap (see figure 2a). The $\beta$ s for the simulations near the chiral transition are not close enough for such an estimate (see figure $\beta$ a). Our (more subjective) estimate for this chiral transition is $\beta_{\chi}=6.85(5)$.

For $\gamma=5$, we estimate the position of the deconfinement transition to be $\beta_{d}=5.420(4)$. For the chiral transition we estimate $\beta_{\chi}=5.450(5)$.

Figures 2.3 show the plaquette distributions near the deconfinement and chiral transitions.

The deconfinement $\beta, \beta_{d}$, is restricted to the range $\beta_{d}(\gamma=\infty) \leq \beta_{d} \leq \beta_{d}\left(\gamma=\gamma_{c}\right)$ with $\beta_{d}(\gamma=$ $\left.\gamma_{c}\right) \leq \beta_{d}$ (quenched). Hence $5.25 \lesssim \beta_{d} \lesssim 5.6925$. [The lower bound (zero 4-fermion coupling) is from [19, 20] while the upper limit (quenched QCD) is from [21, 22].] The chiral-symmetry restoring $\beta, \beta_{\chi}$, is in the range $\beta_{d} \leq \beta_{\chi} \leq \infty$, where the lower bound is achieved for small 4fermion coupling, and the upper for $\gamma=\gamma_{c}$. Table 11 summarises these results. (Note that we have not included error bars on the results of others.) 

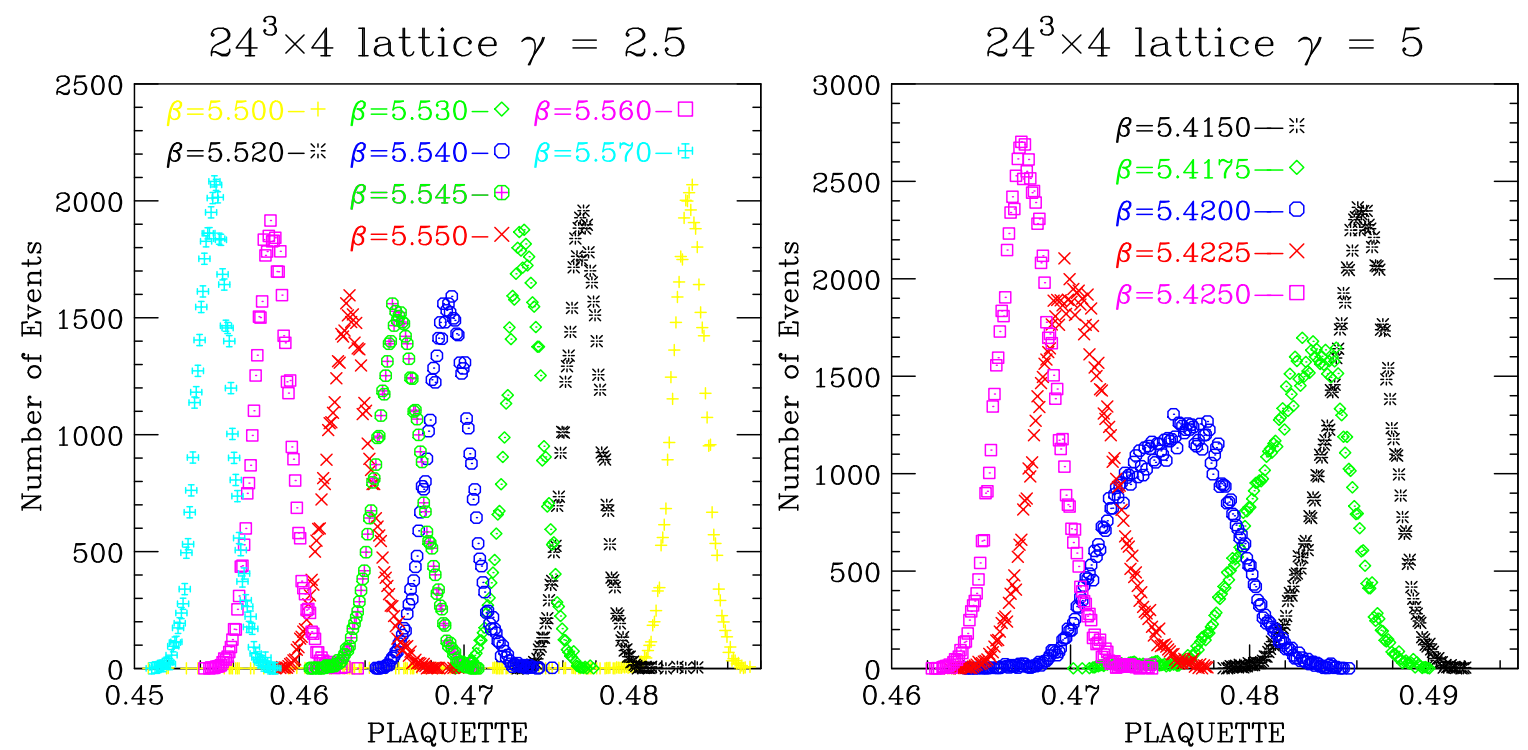

Figure 2: a) Plaquette distributions close to the deconfinement transition on a $24^{3} \times 4$ lattice at $\gamma=2.5$. (Since we have twice the statistics at $\beta=5.545$ as at the other $\beta \mathrm{s}$, we have divided these points by 2). b) Same, but for $\gamma=5$.

\begin{tabular}{lll}
\hline$\gamma$ & $\beta_{d}$ & $\beta_{\chi}$ \\
\hline 0.0 & 5.6925 & - \\
$\gamma_{c} \approx 1.7$ & $?$ & $\infty$ \\
2.5 & $5.547(3)$ & $6.85(5)$ \\
5.0 & $5.420(4)$ & $5.450(5)$ \\
10. & $5.327(2)$ & $5.327(2)$ \\
20. & $5.289(1)$ & $5.289(1)$ \\
$\infty$ & 5.25 & 5.25 \\
\hline
\end{tabular}

Table 1: Deconfinement and chiral-symmetry restoration $\beta$ s as functions of $\gamma$.

\section{Discussion and Conclusions}

- Adding extra 4-fermion interactions with sufficient strength to the lattice QCD action separates the deconfinement and chiral-symmetry restoration transitions at finite temperatures. We are able to change the separation of the scales of confinement and $\chi \mathrm{SB}$ by varying the 4-fermion coupling, as predicted from proposed holographic duals of QCD.

- $5.25 \lesssim \beta_{d} \lesssim 5.6925$, while $\beta_{d} \leq \beta_{\chi} \leq \infty$.

- At $\gamma=2.5-$ strong 4-fermion coupling $-T_{\chi} \sim 10 T_{d}$. At $\gamma=5-$ intermediate 4-fermion coupling $-T_{\chi} \sim 1.04 T_{d}$. For $\gamma \geq 10$ - weak coupling $-T_{\chi}=T_{d}$.

- We should perform simulations at more $\gamma$ values in the range $2 \lesssim \gamma \lesssim 7$. Our simulations used $N_{t}=4$. A more complete study should include other $N_{t}$ values. 

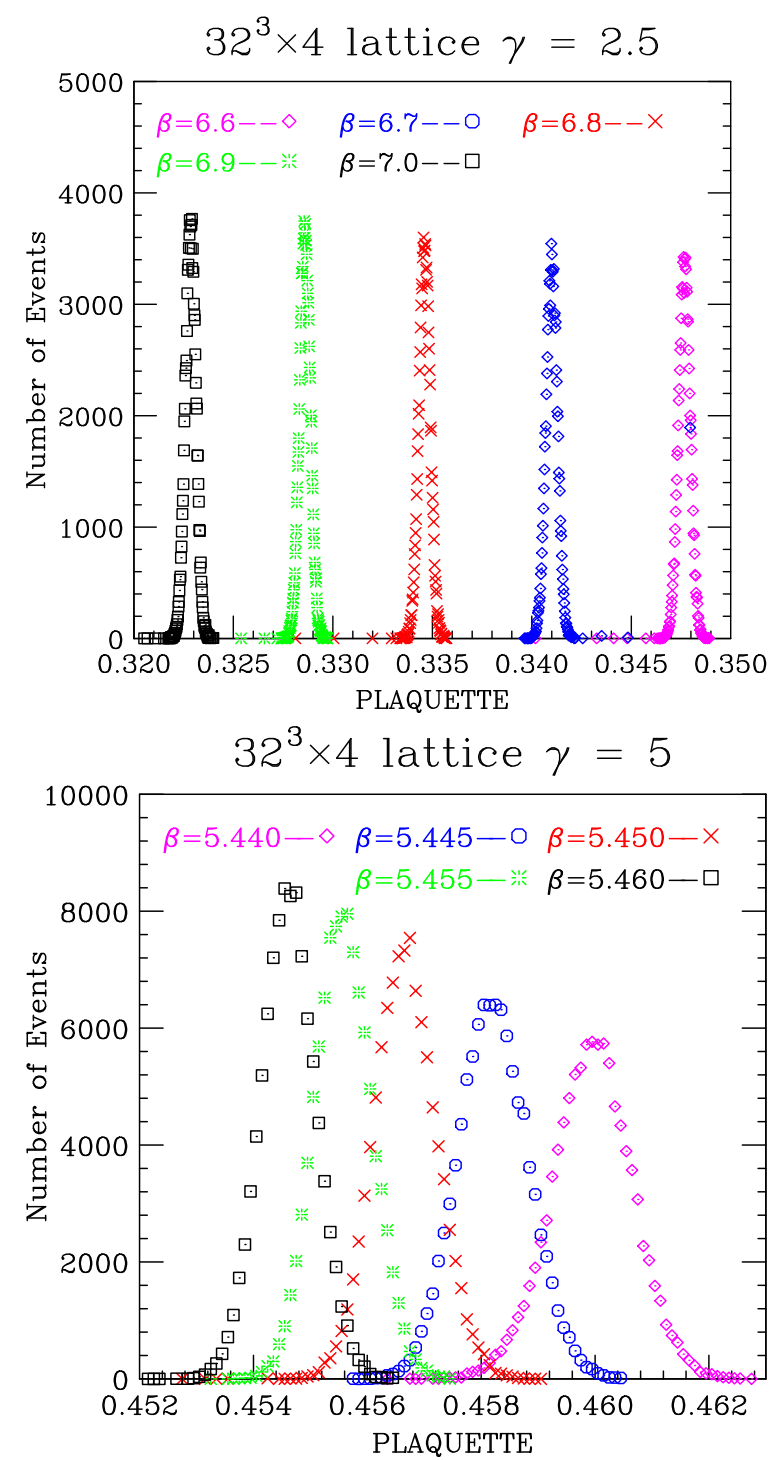

Figure 3: Plaquette distributions close to the chiral-symmetry restoration transition on a $32^{3} \times 4$ lattice (a) at $\gamma=2.5$, (b) at $\gamma=5$.

- We have qualitative agreement with holographic QCD. We need to make the comparison more quantitative.

- Is the deconfinement transition a phase transition, or merely a crossover? As the 4-fermion coupling increases to infinity, we expect the theory to approach quenched QCD, where deconfinement is a first order transition. We also see some indication of this transition becoming stronger as the 4-fermion coupling weakens and the two transitions approach one another.

- With a little more work we should be able to determine the universality class of the second order chiral transition. 
- The two phase transitions appear to coalesce at a finite (non-zero) 4-fermion coupling ( $\gamma$ a little larger than 5).

- We used a local irrelevant 4-fermion interaction, which will not survive the continuum limit. Does the non-local 4-fermion interaction indicated by the AdS/QCD models survive the continuum limit, i.e. does it define a non-perturbatively renormalizable theory? An intermediate model would be a Yukawa model where the auxilliary fields have full 4-dimensional scalar dynamics. Such a model is (or can be) perturbatively renormalizible, but is believed to be non-perturbatively trivial.

- Theories with quarks in higher representations of the colour group, where the stronger QCD coupling separates the confinement and $\chi \mathrm{SB}$ scales even without the extra 4-fermion terms, should be studied. The Bielefeld group have studied adjoint quarks where the transitions are separated and are required by symmetry to both be phase transitions [3]. Colour sextet quarks are also of some interest, especially with regard to conformal technicolor models [23].

\section{Acknowledgements}

These simulations were performed on the Cray XT4, Franklin, at NERSC. Earlier work used the University of Kentucky HP Superdome. We thank Jeffrey Harvey and David Kutasov for raising the questions which inspired this research. We also acknowledge helpful discussions with Cosmas Zachos and Alexander Velytsky.

\section{References}

[1] J. Polonyi, H. W. Wyld, J. B. Kogut, J. Shigemitsu and D. K. Sinclair, Phys. Rev. Lett. 53, 644 (1984).

[2] J. B. Kogut, J. Shigemitsu and D. K. Sinclair, Phys. Lett. B 145, 239 (1984).

[3] J. Engels, S. Holtmann and T. Schulze, Nucl. Phys. B 724, 357 (2005) [arXiv:hep-lat/0505008].

[4] A. Karch and E. Katz, JHEP 0206, 043 (2002) [arXiv:hep-th/0205236].

[5] T. Sakai and S. Sugimoto, Prog. Theor. Phys. 113, 843 (2005) [arXiv:hep-th/0412141].

[6] T. Sakai and S. Sugimoto, Prog. Theor. Phys. 114, 1083 (2006) [arXiv:hep-th/0507073].

[7] E. Antonyan, J. A. Harvey, S. Jensen and D. Kutasov, arXiv:hep-th/0604017.

[8] O. Aharony, J. Sonnenschein and S. Yankielowicz, Annals Phys. 322, 1420 (2007) [arXiv:hep-th/0604161].

[9] T. Banks and A. Casher, Nucl. Phys. B 169, 103 (1980).

[10] H. Leutwyler and A. Smilga, Phys. Rev. D 46, 5607 (1992).

[11] D. J. Gross and A. Neveu, Phys. Rev. D 10, 3235 (1974).

[12] Y. Nambu and G. Jona-Lasinio, Phys. Rev. 122, 345 (1961).

[13] Y. Nambu and G. Jona-Lasinio, Phys. Rev. 124, 246 (1961).

[14] D. K. Sinclair, arXiv:0805.4627 [hep-lat]. 
[15] J. B. Kogut, J. F. Lagaë and D. K. Sinclair, Phys. Rev. D 58, 034504 (1998) [arXiv:hep-lat/9801019].

[16] M. A. Clark and A. D. Kennedy, Phys. Rev. D 75, 011502 (2007) [arXiv:hep-lat/0610047].

[17] J. B. Kogut and D. K. Sinclair, arXiv:hep-lat/0211008.

[18] A. M. Ferrenberg and R. H. Swendsen, Phys. Rev. Lett. 61, 2635 (1988).

[19] C. W. Bernard et al., Phys. Rev. D 61, 054503 (2000) [arXiv:hep-lat/9908008].

[20] M. D’Elia, A. Di Giacomo and C. Pica, Phys. Rev. D 72, 114510 (2005) [arXiv:hep-lat/0503030].

[21] F. R. Brown, N. H. Christ, Y. F. Deng, M. S. Gao and T. J. Woch,

[22] P. Bacilieri et al., Phys. Rev. Lett. 61, 1545 (1988).

[23] Y. Shamir, B. Svetitsky and T. DeGrand, arXiv:0803.1707 [hep-lat]. 